\title{
Raman spectroscopic Stratification of Multiple Myeloma patients based on Exosomes profiling
}

Mario Russo ${ }^{1 \#, ~ L u c a ~ T i r i n a t o ~}{ }^{1 \#}$, Francesca Scionti², Maria Laura Coluccio ${ }^{1}$, Gerardo Perozziello ${ }^{1}$, Caterina Riillo², Vincenzo Mollace $^{3}$, Santo Gratteri ${ }^{3}$, Natalia Malara ${ }^{1}$, Maria Teresa Di Martino², Giuseppe Viglietto², Pierosandro Tagliaferri ${ }^{2}$, Pierfrancesco Tassone ${ }^{2}$, Marco Rossi ${ }^{2 *}$ and Patrizio Candeloro ${ }^{1 *}$

1. BioNEM (Bio and Nano Engineering for Medicine) Laboratory, Dipartimento di Medicina Sperimentale e Clinica, Università Magna Graecia, 88100, Catanzaro, Italy.

2. Dipartimento di Medicina Sperimentale e Clinica, Università Magna Graecia, 88100, Catanzaro, Italy.

3. Institute of Research of Food Safety \& Health (IRC-FSH), Dipartimento di Scienza della Salute, Università Magna Graecia, 88100, Catanzaro, Italy.

\# These authors equally contributed to the present work

* Corresponding auhtors. Email addresses: patrizio.candeloro@unicz.it (P. Candeloro); rossim@unicz.it (M. Rossi)

\section{Figure S1 - Raman spectrum of Exosome Resuspension buffer}

Raman spectrum of Exosome Resuspension Buffer has been measured in order to investigate potential interferences with Raman spectra of Exosome samples from patients.

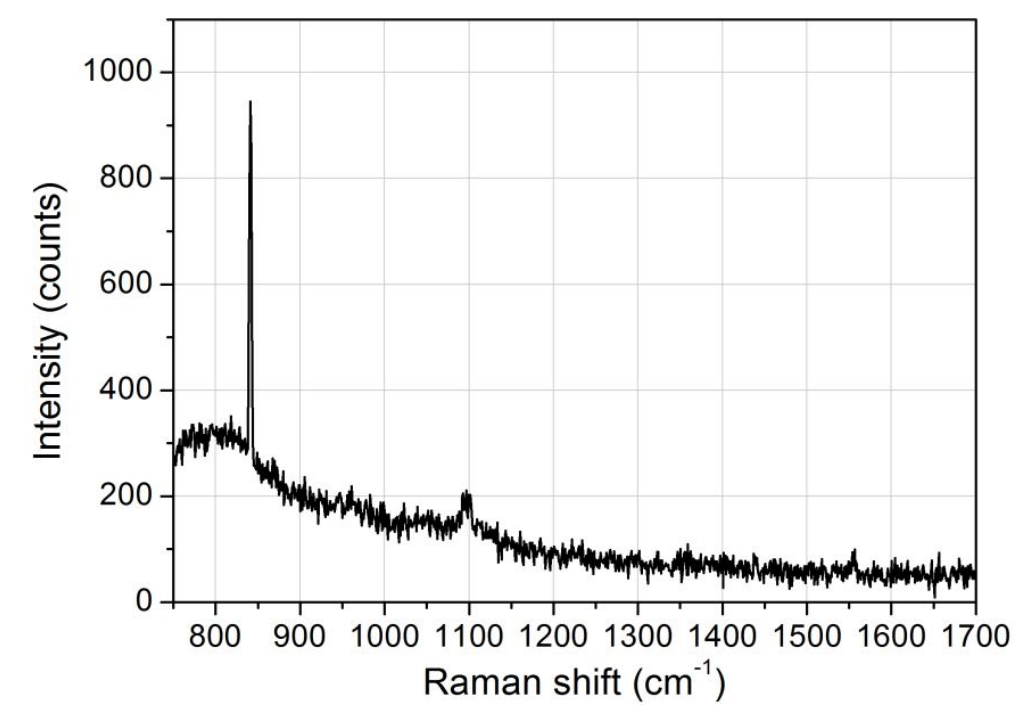

Figure S1: The buffer present in the miRCURY ${ }^{\mathrm{TM}}$ Exosome Isolation Kit and used for the exosome resuspension has been measured and its spectrum is here reported. Negligible Raman signal has been detected, except for a sharp peak at $860 \mathrm{~cm}^{-1}$ which is however easily detectable in Raman measurements of exosomes. 


\section{Table S1 - Patients list}

List of all patients from which exosomes have been extracted. Patient sex, neoplastic stage and age are reported too. The color code is in agreement with the colors reported in the main text.

\begin{tabular}{|llll|}
\hline ID Sample & Stage & Sex & Age (years) \\
\hline CP & MGUS & M & 81 \\
LGP1 & MGUS & M & 45 \\
1 CA & MGUS & M & 80 \\
2 CB & MGUS & M & 54 \\
$22 \mathrm{CN}$ & MGUS & F & 76 \\
24 GC & MGUS & M & 61 \\
38 SF & MGUS & F & 51 \\
48 CR & MGUS & F & 53 \\
59 SF & MGUS & F & 51 \\
8 VV & MGUS & M & 58 \\
$12 \mathrm{BT}$ & MGUS & F & 63 \\
36 DD & aMM & M & 67 \\
35 CL & aMM & F & 73 \\
11 VS & aMM & F & 55 \\
$10 \mathrm{CD}$ & aMM & F & 54 \\
IG & sMM & M & 73 \\
MF & sMM & F & 48 \\
FI & sMM & F & 81 \\
AR & sMM & F & 70 \\
SF & sMM & F & 75 \\
MC & sMM & F & 81 \\
DLM & sMM & F & 61 \\
PR & sMM & M & 71 \\
ZF & sMM & M & 69 \\
EM & sMM & F & 67 \\
BD & sMM & M & 83 \\
FF & sMM & M & 69 \\
DA & sMM & M & 90 \\
\hline
\end{tabular}




\section{Figure S2 - Loading curve of rotated PC2}

Rotated-PC2 loads, calculated after the rotation of the Principal Components in the PC1-PC2 plane.

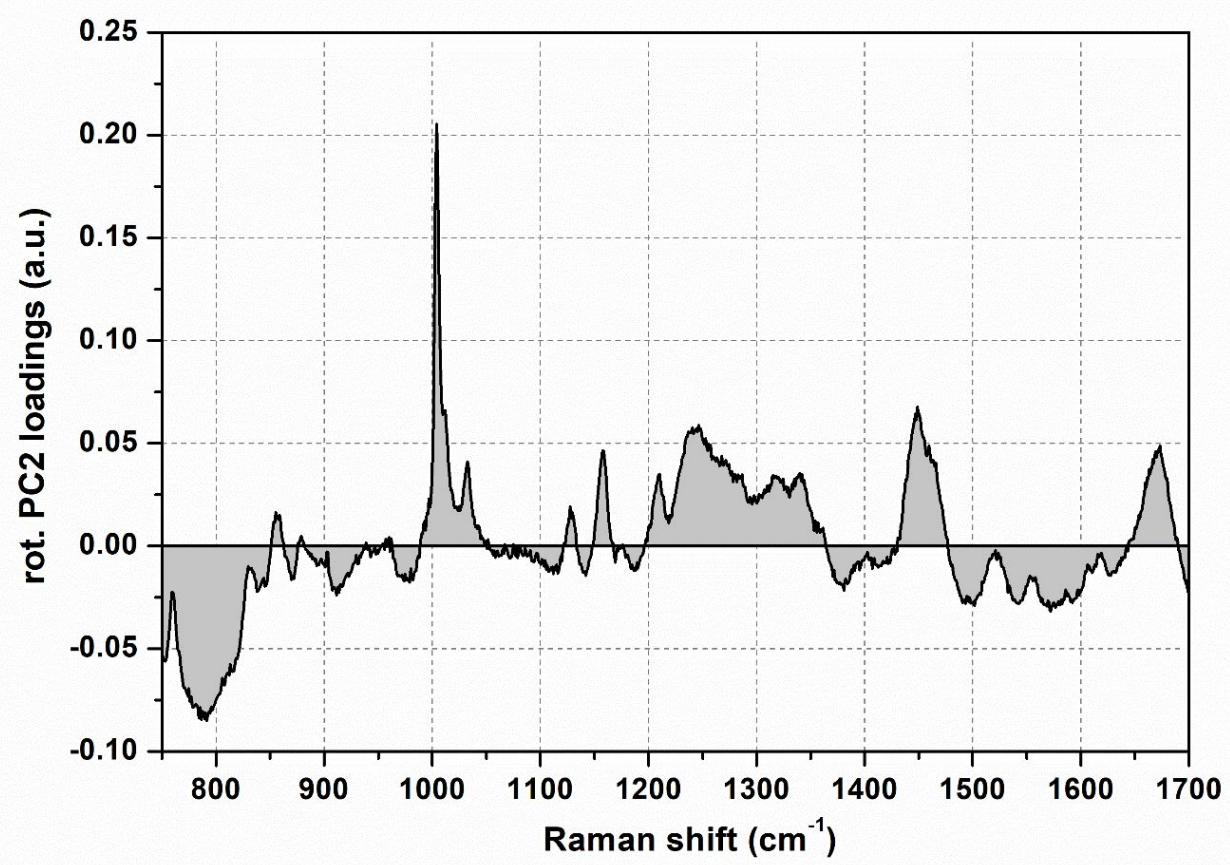

Figure S2: Rot.PC2 loading spectrum. It is a $32^{\circ}$ counterclockwise rotated PC2 that is able, solely, to discriminate among all the exosome groups. The assignment of most important peaks can be found in the Table S2. 


\section{Table S2 - Assignment of the main peaks of the rot.PC2 loadings}

Biochemical assignment of the main important peaks present in the rot.PC2 loading spectrum (Fig.S2 above). For the References of SI please see at the end of this document.

\begin{tabular}{|l|l|l|}
\hline $\begin{array}{l}\text { Raman Shift } \\
\left(\mathrm{cm}^{-1}\right)\end{array}$ & Tentative Peak Assignment & $\begin{array}{l}\text { Reference in } \\
\text { SI }\end{array}$ \\
\hline 787 & Nucleic Acids & {$[1]$} \\
$850-860$ & Polysaccharide Structure & {$[2]$} \\
1003 & Phenylalanine & {$[2]$} \\
1032 & Phospholipid and/or Polysaccharide & {$[3]$} \\
1128 & Proteins and/or Ceramides & {$[2]$} \\
1157 & $\beta$-Carotene Accumulation & {$[1]$} \\
1209 & Tryptophan and/or Phenylalanine & {$[1]$} \\
$1235-1285$ & Proteins and/or Nucleic Acids and/or Lipids & {$[3]$} \\
1316 & Nucleic Acids and/or Collagen and/or Guanine & {$[4]$} \\
$1335-1345$ & $\begin{array}{l}\text { Nucleic Acids (Purine Bases) and/or Tryptophan } \\
\text { and/or Glycine Backbone and/or Proline Side }\end{array}$ & {$[2]$} \\
1378 & $\begin{array}{l}\text { Chain } \\
\text { Carbohydrate and/or Nucleic Acids }\end{array}$ & {$[4]$} \\
$1440-1450$ & Proteins and/or Lipids & {$[5]$} \\
$1490-1500$ & Nucleic Acids & {$[1]$} \\
1539 & Nucleic Acids & {$[6]$} \\
$1560-1580$ & $\begin{array}{l}\text { Tryptophan and/or Nucleic Acids and/or Proteins } \\
\text { and/or Carbohydrates }\end{array}$ & {$[7]$} \\
1672 & Ceramide & {$[1]$} \\
\hline
\end{tabular}

Table S2: Tentative Raman assignment, from literature, of the main peaks in rot.PC2 loadings. In red are reported the peaks downregulated in the sMM EX population, while in black the upregulated ones. 


\section{Figure S3 - SERS substrates}

Scanning electron microscopy (SEM) picture of SERS substrates

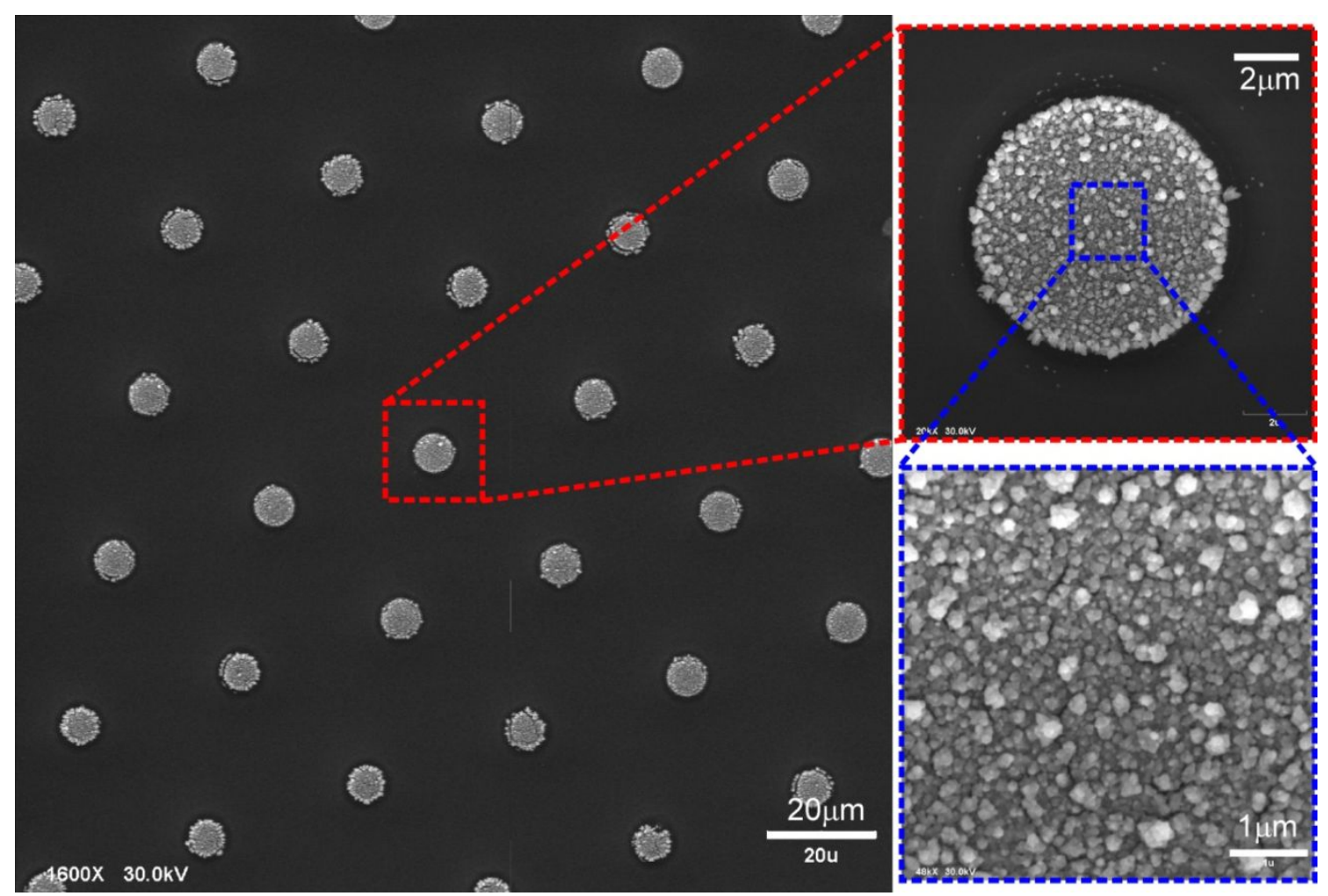

Figure S3: Scanning electron microscopy of SERS substrates. Microdisks containing Au nanoparticles are fabricated by means of optical lithography and Au electroless deposition. 


\section{Figure S4 - Raman spectra recorded with $633 \mathrm{~nm}$ and $830 \mathrm{~nm}$ excitation wavelengths}

Comparison between standard Raman spectra recorded with $633 \mathrm{~nm}$ and $830 \mathrm{~nm}$ excitation wavelengths, in order to investigate potential wavelength-dependent effects.

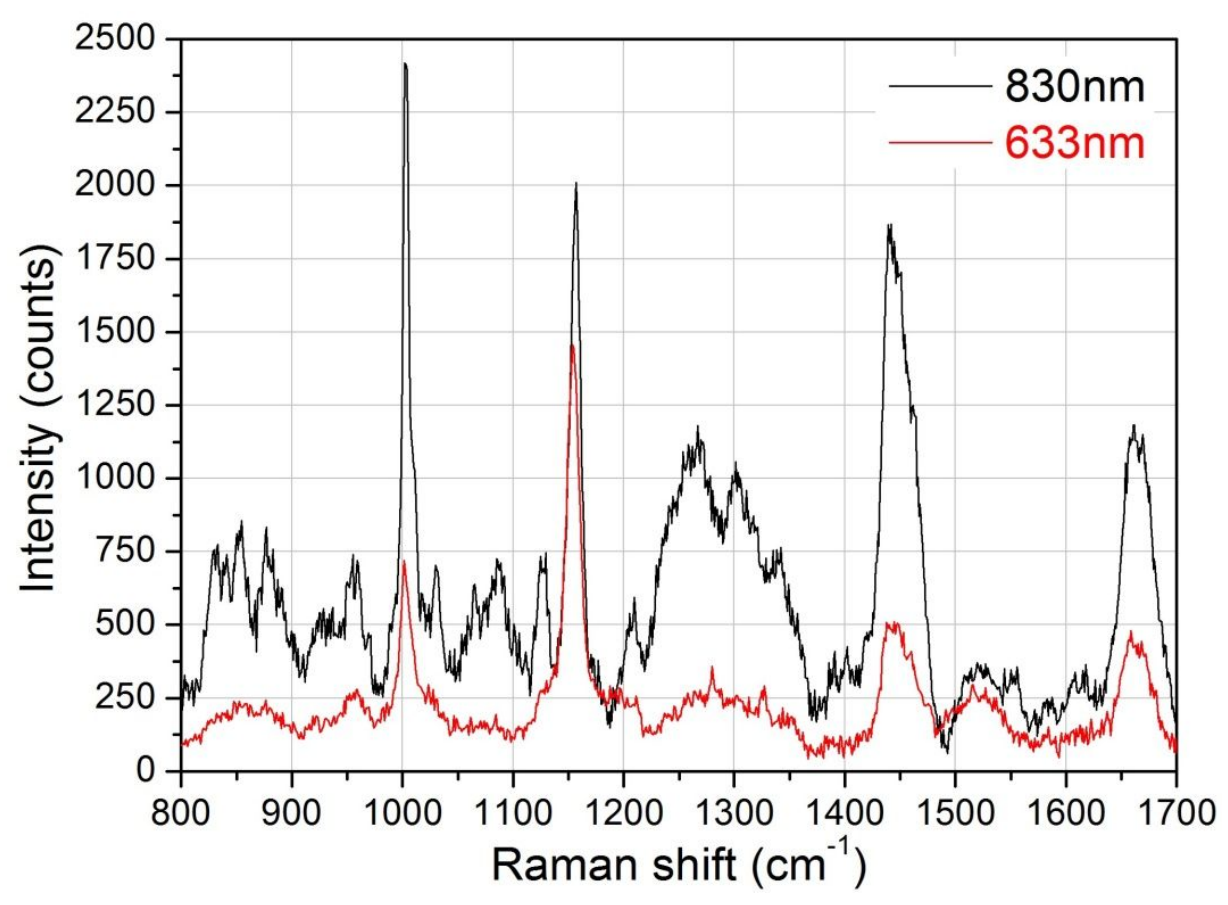

Figure S4: Raman spectra recorded on one of the Multiple Myeloma derived exosomes samples using two different excitation sources at $633 \mathrm{~nm}$ and $830 \mathrm{~nm}$. It can be observed that all the major Raman peaks/bands are the same for the two sources, thus proving that no wavelegnth-dependent effects are present. Moreover some minor spectral features can be better appreciated with the $830 \mathrm{~nm}$ laser, mainly due to the smaller fluorescence effects arising with IR sources. 


\section{References of SI}

1 Movasaghi, Z.; Rehman, S.; Rehman, I. U., Raman spectroscopy of biological tissues. Appl. Spectrosc. Rev. 2007, 42, (5), 493-541.

2 Gualerzi, A.; Niada, S.; Giannasi, C.; Picciolini, S.; Morasso, C.; Vanna, R.; Rossella, V.; Masserini, M.; Bedoni, M.; Ciceri, F.; Bernardo, M. E.; Brini, A. T.; Gramatica, F., Raman spectroscopy uncovers biochemical tissue-related features of extracellular vesicles from mesenchymal stromal cells. Sci. Rep. 2017, 7, 9820.

3 Stremersch, S.; Marro, M.; Pinchasik, B. E.; Baatsen, P.; Hendrix, A.; De Smedt, S. C.; Loza-Alvarez, P.; Skirtach, A. G.; Raemdonck, K.; Braeckmans, K., Identification of Individual Exosome-Like Vesicles by Surface Enhanced Raman Spectroscopy. Small 2016, 12, (24), 3292-3301.

4 Zhang, P.; Wang, L. M.; Fang, Y. P.; Zheng, D. W.; Lin, T. F.; Wang, H. Q., Label-Free Exosomal Detection and Classification in Rapid Discriminating Different Cancer Types Based on Specific Raman Phenotypes and Multivariate Statistical Analysis. Molecules 2019, 24, (16), 2947.

5 Yanez-Mo, M.; Siljander, P. R. M.; Andreu, Z.; Zavec, A. B.; Borras, F. E.; Buzas, E. I.; Buzas, K.; Casal, E.; Cappello, F.; Carvalho, J.; Colas, E.; Cordeiro-da Silva, A.; Fais, S.; Falcon-Perez, J. M.; Ghobrial, I. M.; Giebel, B.; Gimona, M.; Graner, M.; Gursel, I.; Gursel, M.; Heegaard, N. H. H.; Hendrix, A.; Kierulf, P.; Kokubun, K.; Kosanovic, M.; KraljIglic, V.; Kramer-Albers, E. M.; Laitinen, S.; Lasser, C.; Lener, T.; Ligeti, E.; Line, A.; Lipps, G.; Llorente, A.; Lotvall, J.; Mancek-Keber, M.; Marcilla, A.; Mittelbrunn, M.; Nazarenko, I.; Nolte-t' Hoen, E. N. M.; Nyman, T. A.; O'Driscoll, L.; Olivan, M.; Oliveira, C.; Pallinger, E.; del Portillo, H. A.; Reventos, J.; Rigau, M.; Rohde, E.; Sammar, M.; SanchezMadrid, F.; Santarem, N.; Schallmoser, K.; Ostenfeld, M. S.; Stoorvogel, W.; Stukelj, R.; Van der Grein, S. G.; Vasconcelos, M. H.; Wauben, M. H. M.; De Wever, O., Biological properties of extracellular vesicles and their physiological functions. J. Extracell. Vesicles 2015, 4, 27066.

6 Prescott, B.; Steinmetz, W.; Thomas, G. J., Jr., Characterization of DNA structures by laser Raman spectroscopy. Biopolymers 1984, 23, (2), 235-56.

7 Yan, Z. B.; Dutta, S.; Liu, Z. R.; Yu, X. K.; Mesgarzadeh, N.; Ji, F.; Bitan, G.; Xie, Y. H., A Label-Free Platform for Identification of Exosomes from Different Sources. ACS Sens. 2019, 4, (2), 488-497. 